\title{
REGULAR GLEASON MEASURES AND GENERALIZED EFFECT ALGEBRAS
}

\author{
ANATOLIJ DVUREČENSKIJ ${ }^{1,2}$, JIŘÍ JANDA ${ }^{3}$ \\ 11 Mathematical Institute, Slovak Academy of Sciences, \\ Štefánikova 49, SK-814 73 Bratislava, Slovakia \\ 2 Depart. Algebra Geom., Palacký Univer. \\ 17. listopadu 12, CZ-771 46 Olomouc, Czech Republic \\ ${ }^{3}$ Depart. Math. and Statistics, Faculty of Science \\ Masaryk University, Kotlářská 267/2, CZ-611 37 Brno, Czech Republic \\ E-mail: dvurecen@mat.savba.sk xjanda@math.muni.cz
}

\begin{abstract}
We study measures, finitely additive measures, regular measures, and $\sigma$-additive measures that can attain even infinite values on the quantum logic of a Hilbert space. We show when particular classes of non-negative measures can be studied in the frame of generalized effect algebras.
\end{abstract}

\section{Introduction}

A basic theoretical tool of quantum mechanical measurements is based on the famous Gleason Theorem Gle which says that if $H$ is a separable Hilbert space over real, complex or quaternion numbers, $\operatorname{dim} H \neq 2$, then there is a one-toone correspondence between $\sigma$-additive states, $m$, on the system $\mathcal{L}(H)$ of all closed subspaces of $H$, and the set of von Neumann operators on $H$, i.e. positive Hermitian operators, $T$, of trace equal to 1 , given by

$$
m(M)=\operatorname{tr}\left(T P_{M}\right), \quad M \in \mathcal{L}(H),
$$

where $P_{M}$ is an orthogonal projector onto $M$. Formula (1.1) holds also for any completely additive state for every $\mathcal{L}(H), \operatorname{dim} H \neq 2$. For an extension of the Gleason Theorem to non-separable Hilbert spaces, see e.g. Dvu2, Dvu3. In such a cases, if $\operatorname{dim} H \neq 2$ is a non-measurable cardinal, then every $\sigma$-additive state is completely additive. In addition, formula (1.1) is valid also for bounded signed $\sigma$-additive measures, Dvu3.

However, there are also important measures attaining even infinite values, like, $\operatorname{dim} M, M \in \mathcal{L}(H)$, or attaining both positive and negative values, e.g. $\operatorname{tr}\left(T\left(A P_{M}+\right.\right.$ $\left.\left.P_{M} A\right) / 2\right), M \in \mathcal{L}(H)$, where $A$ is a Hermitian operator on $H$ that is neither positive nor negative. Also for them it is possible to find an extension of the Gleason formula

\footnotetext{
${ }^{1}$ Keywords: Hilbert space, measure, regular measure, $\sigma$-additive measure, Gleason measure, generalized effect algebra, bilinear form, singular bilinear form, regular bilinear form, monotone convergence

AMS classification: 81P15, 03G12, 03B50

The authors acknowledge the support (A.D.) by the Slovak Research and Development Agency under the contract APVV-0178-11, the grant VEGA No. 2/0059/12 SAV, ESF Project CZ.1.07/2.3.00/20.0051, (J.J.) ESF Project CZ.1.07/2.3.00/20.0051 and Masaryk University grant 0964/2009
} 
(1.1), however, in such a case, they are induced rather by bilinear forms than by trace operators, see e.g. [LuSh] as it will be shown by formula (3.1). Therefore, we call Gleason measures all measures that can be expressed by a generalized Gleason formula.

If we study finitely measures, $m$, with improper values, then regularity of $m$, i.e. the property "given $M \in \mathcal{L}(H)$, the value $m(M)$ can be approximated by values of $m(N)$, where $N$ is a finite-dimensional subspace of $M$ ", does not implies that the measure is $\sigma$-additive as in the case that $m$ is a finite measure. By Dvu3, Lem 3.7.8. Thm 3.7.9], every positive regular measure $m$ that is $\sigma$-finite (i.e. $H$ can be split into a sequence of mutually orthogonal subspaces of finite measure) is in a one-to-one correspondence with densely defined positive bilinear forms. Consequently, regular measures are Gleason measures, too. Since, not every bilinear form is induced by a Hermitian operator, bilinear forms define more regular measures than do bilinear forms induced by Hermitian trace operators via (1.1). For more details on measures on $\mathcal{L}(H)$, we recommend to consult the book Dvu3].

Foulis and Bennett FoBe have introduced effect algebras that are inspired by mathematical models of quantum mechanics. They model both a two-valued reasoning and a many valued one. They have a least and a top element, 0 and 1, respectively, and a basic operation is a partially defined addition + . For example, POV-measures have within these models a natural form as observables. More general structures than effect algebras are generalized effect algebras [DvPu], which have a similar partially defined addition, but the top element 1 is not guaranteed. For additional information on effect algebras and generalized effect algebras, we recommend the monograph [Dvu3].

The aim of this paper is to study some properties of measures using methods of generalized effect algebras. We describe a structure of generalized effect algebras of finitely additive Gleason measures, regular measures, and $\sigma$-measures. In addition, we study also questions connected with a Dedekind monotone $\sigma$-convergence.

The paper is organized as follows. Section 2 presents elements of bilinear forms on a Hilbert space and frame functions that are semibounded. Regular measures and their properties are studied in Section 3 together with different kinds of generalized effect algebras. In Section 4, we deal with monotone convergence properties of $\sigma$-additive measures.

\section{Bilinear Forms, Signed Measures, and Frame Functions}

Let $S$ be a real or complex inner product space with an inner product $(\cdot, \cdot)$ which is linear in the left argument and antilinear in the right one. Sometimes we call $S$ a pre-Hilbert space. If $S$ is complete with respect to the norm $\|\cdot\|$ induced by the inner product, $S$ is said to be a Hilbert space. Let $H$ be a Hilbert space and let $\mathcal{L}(H)$ be the system of all closed subspaces of $H$. We denote by $\mathbf{0}$ the zero vector of $H$ and if $x_{1}, \ldots, x_{n} \in H, \operatorname{sp}\left(x_{1}, \ldots, x_{n}\right)$ denotes the span generated by $x_{1}, \ldots, x_{n}$. We denote by $\mathcal{S}(H)$ the unit sphere of $H$. Similarly, if $S$ is a linear submanifold of $H, \mathcal{S}(S)$ denotes the unit sphere of $S$.

Any system of mutually orthogonal unit vectors $\left\{x_{i}\right\}$ of an inner product space $S$ is denoted as ONS. An ONS $\left\{x_{i}\right\}$ is said to be (i) a maximal ONS (MONS for short) if $x$ is a vector orthogonal to each vector $x_{i}$, then $x=\mathbf{0}$; (ii) orthonormal base (ONB for short), if for any vector $x \in S$, we have $x=\sum_{i}\left(x, x_{i}\right) x_{i}$. 
A Hermitian operator $T$ on $H$ is said to be a trace operator (or of finite trace) if there is a real constant $\operatorname{tr}(T)$, called the trace of $T$, such that for any orthonormal basis $\left\{x_{i}\right\}, \operatorname{tr}(T)=\sum_{i}\left(T x_{i}, x_{i}\right)$. We denote by $\operatorname{Tr}(H)$ the class of Hermitian operators with finite trace.

Now we introduce bilinear forms. Let $D(t)$ be a submanifold of a complex Hilbert space $H$ and let $D(t)$ be dense in $H$. A mapping $t: D(t) \times D(t) \rightarrow \mathbb{C}$ is called a bilinear form (or a sesquilinear form) if and only if it is additive in both arguments and $(\alpha x, \beta y)=\alpha \bar{\beta}(x, y)$ for all $\alpha, \beta \in \mathbb{C}, x, y \in D(t)$, where $\bar{\beta}$ is the complex conjugation of $\beta$. A complex-valued function $\hat{t}$ with the definition domain $D(t)$ is defined by $\hat{t}(x)=t(x, x), x \in D(t)$, and it is said to be a quadratic form induced by the bilinear form $t$, [Hal, p. 12]. A bilinear form $t$ is called symmetric if $t(x, y)=\overline{t(y, x)}$.

A symmetric bilinear form $t$ is (i) semibounded if $\inf \{t(x, x): x \in \mathcal{S}(D(t))\}>$ $-\infty$, (ii) positive if $t(x, x) \geq 0, x \in \mathcal{S}(D(t))$, and (iii) bounded if $\sup \{|t(x, x)|$ : $x \in \mathcal{S}(D(t))\}<\infty$; otherwise $t$ is called unbounded. We denote by $\mathcal{B F}(H)$ and $\mathcal{P B F}(H)$ the set of all bilinear forms and positive bilinear forms, respectively, on $H$. We denote by $o$ the bilinear with $D(o)=H$ defined by $o(x, y)=0$ for all $x, y \in H$; then $o \in \mathcal{P B F}(H)$.

On $\mathcal{B F}(H)$, we can define a usual sum $t+s$ for each $t, s$ on $D(t+s):=D(t) \cap D(s)$ by $(t+s)(x, y):=t(x, y)+s(x, y)$ for all $x, y \in D(t) \cap D(s)$, and the multiplication by a scalar $\alpha \in \mathbb{C}$ by $(\alpha t)(x, y):=\alpha t(x, y)$ for $x, y \in D(\alpha t):=D(t)$.

Given two densely defined positive bilinear forms $t$ and $s$, we write

$$
t \preceq s
$$

if and only if $D(t) \supseteq D(s)$ and $t(x, x) \leq s(x, x)$ for all $x \in D(s)$. Then $\preceq$ is a partial order on $\mathcal{P B F}(\mathcal{H})$, and the bilinear form $o$ is the least element of the set $\mathcal{P B F}(\mathcal{H})$, i.e. $o \preceq t$ for any $t \in \mathcal{P B \mathcal { F }}(\mathcal{H})$.

A bilinear form $s$ is an extension of a bilinear form $t$ iff $D(t) \subseteq D(s)$ and, for every $x, y \in D(t), t(x, y)=s(x, y)$; we will write $s_{\mid D(t)}=t$. A bilinear form $t$ is closable if it has some closed extension. Then the least closed extension of a closable $t$ is said to be a closure and we denote it by $\bar{t}$.

For any densely defined operator $A$ with domain $D(A)$ on $H$, the mapping $t(x, y):=(A x, y)$ is a bilinear form on $D(A)$. We say that a bilinear form $t$ corresponds to an operator $A$ iff $D(A) \subseteq D(t)$ and, for every $x, y \in D(A), t(x, y)=$ $(A x, y)$. We say that $t$ is generated by $A$ if $t$ corresponds to $A$ and $D(A)=D(t)$. We note that there are bilinear forms that are not generated by any operator. In addition, there is a one-to-one correspondence between closed positive symmetric bilinear forms, $t$, and positive self-adjoint operators, $A$, given by $D(t)=D\left(A^{1 / 2}\right)$, and $t(x, y)=\left(A^{1 / 2} x, A^{1 / 2} y\right), x, y \in D(t)$.

Given a symmetric semibounded bilinear form $t$, we can equip its domain $D(t)$ with an inner product $(x, y)_{t}:=t(x, y)+\left(1+m_{t}\right)(x, y)$. In this way $D(t)$ becomes a pre-Hilbert space. Whenever $D(t)$ with $(x, y)_{t}$ is a Hilbert space, we call $t$ closed.

A bilinear form $s$ is an extension of a bilinear form $t$ iff $D(t) \subseteq D(s)$ and, for every $x, y \in D(t), t(x, y)=s(x, y)$; we will write $s_{\mid D(t)}=t$. A bilinear form $t$ is closable if it has some closed extension.

An important result which is also a matter of interest for us was proved in [Sim, Thm 2.1, Thm 2.2]: 
Theorem 2.1. Let $t$ be a densely defined positive symmetric bilinear form on a Hilbert space $H$. Then there exist two positive symmetric bilinear forms $t_{r}$ and $t_{s}$ such that $D(t)=D\left(t_{r}\right)=D\left(t_{s}\right)$ such that

$$
t=t_{r}+t_{s}
$$

where $t_{r}$ is the largest closable bilinear form less than $t$ in the ordering $\preceq$.

In view of Theorem 2.1. the components $t_{r}$ and $t_{s}$ of the positive symmetric bilinear form $t$, are said to be the regular and singular part of $t$, respectively.

If $t_{s}$ is identical zero, the bilinear form $t$ is said to be regular, and if $t_{r}$ is identical zero, $t$ is said to be singular. Hence, the bilinear form $o$ is the least positive symmetric bilinear form and is a unique positive symmetric bilinear form that is simultaneously regular and singular.

The following example presents a nonzero everywhere defined positive singular symmetric bilinear form.

Example 2.2. Let $\left\{e_{n}\right\}$ be an orthonormal basis of an infinite-dimensional Hilbert space $H$ which is a part of a Hamel basis $\left\{e_{i}\right\}_{i \in I}$ of $H$ consisting of unit vectors from $H$. Fix an element $e_{i_{0}}, i_{0} \in I$, which does not belong to the orthonormal basis $\left\{e_{n}\right\}$, and define a linear operator $T$ on $H$ by

$$
T\left(\sum_{i} \alpha_{i} e_{i}\right)=\alpha_{i_{0}} e_{i_{0}}
$$

where $\alpha_{i_{0}}$ is the scalar corresponding to $e_{i_{o}}$ in the decomposition $x=\sum_{i} \alpha_{i} e_{i}$, $x \in H$, with respect to the given Hamel basis. Then $T$ is an everywhere defined unbounded linear operator.

If we define a bilinear form $t$ with $D(t)=H$ via

$$
t(x, y)=(T x, T y), x, y \in H,
$$

then $t$ is a nonzero everywhere defined positive singular symmetric bilinear form.

A mapping $m: \mathcal{L}(H) \rightarrow[-\infty, \infty]$ is said to be a finitely additive signed measure if (i) $m(\operatorname{sp}(\mathbf{0}))=0$, and (ii) $m(M \vee N)=m(M)+m(N)$ whenever $M \perp N$, $M, N \in \mathcal{L}(H)$. We note that $m$ can attain from the improper values $\{-\infty, \infty\}$ at most one value.

If for $m: \mathcal{L}(H) \rightarrow[-\infty, \infty]$ with the above (i) and instead of (ii) we have (ii)' $m\left(\bigvee_{i \in I} M_{i}\right)=\sum_{i \in I} m\left(M_{i}\right)$ whenever $M_{i} \perp M_{j}$ for $i \neq j, i, j \in I$ holds for any index set $I, m$ is said to be a completely additive signed measure (also totally additive); if it holds only for any countable index set $I, m$ is said to be a $\sigma$-additive signed measure. If $m(M) \geq 0$ for any $M \in \mathcal{L}(H)$, we are speaking on a finitely additive measure, a completely additive measure, and a $\sigma$-additive measure, respectively. Every finitely additive measure is monotone. If, in addition, $m(M) \geq$ 0 for any $M \in \mathcal{L}(H)$ and $m(H)=1, m$ is said to be a finitely additive state, a completely additive measure and a $\sigma$-additive state, respectively. As we have already mentioned in Introduction, if $m$ is a completely additive state on $\mathcal{L}(H)$, $\operatorname{dim} H \neq 2$, then by the Gleason Theorem, see e.g. [Dvu3, Thm 3.2.21], there is a unique positive Hermitian operator $T$ on $H$ with $\operatorname{trace} \operatorname{tr}(T)=1$ such that

$$
m(M)=\operatorname{tr}\left(T P_{M}\right), \quad M \in \mathcal{L}(H) .
$$

If $H$ is a finite-dimensional Hilbert space with $\operatorname{dim} H \neq 2$ and $m$ is a finite finitely additive signed measure such that $\inf \{m(\operatorname{sp}(x)): x \in \mathcal{S}(H)\}>-\infty$, then $m$ is 
bounded and there is a unique Hermitian operator $T$ on $H$ such that (2.1) holds, see [She, Dvu1] or [Dvu3, Thm 3.2.16].

A finitely additive measure $m$ is $\sigma$-finite if there is a sequence $\left\{M_{i}\right\}$ of mutually orthogonal subspaces of $H$ such that $\bigvee_{i} M_{i}=H$ and every $m\left(M_{i}\right)$ is finite.

A function $f: \mathcal{S}(H) \rightarrow[-\infty, \infty]$ is said to be a frame function if, for any $M \in \mathcal{L}(H)$, there is a constant $W_{M} \in[-\infty, \infty]$ such that $\sum_{i} f\left(x_{i}\right)=W_{M}$ holds for any ONB $\left\{x_{i}\right\}$ in $M$. The constant $W_{M}$ is said to be the weight of $f$ on $M \in \mathcal{L}(H)$. It is worthy of recalling that (i) if $W_{H}=\infty$, then $W_{M}>-\infty$ for any $M \in \mathcal{L}(H)$, (ii) if $W_{H}=-\infty$, then $W_{M}<\infty$ for any $M \in \mathcal{L}(H)$. In what follows, we will study frame functions $f$ such that $f(x)>-\infty$ for any $x \in \mathcal{S}(H)$.

We note that if $f: \mathcal{S}(H) \rightarrow \mathbb{R}$ has the property that there is a constant $W \in \mathbb{R}$ such that $\sum_{i} f\left(x_{i}\right)=W$ for any ONB $\left\{x_{i}\right\}$, then $f$ is a frame function. Indeed, if $M \in \mathcal{L}(H)$ is given, take two ONB's $\left\{x_{i}\right\}$ and $\left\{y_{i}\right\}$ of $M$ and let $\left\{z_{j}\right\}$ be an ONB in $M^{\perp}$. Then $\left\{x_{i}\right\} \cup\left\{z_{j}\right\}$ and $\left\{y_{i}\right\} \cup\left\{z_{j}\right\}$ are ONBs of $H$. Hence, $\sum_{i} f\left(x_{i}\right)+$ $\sum_{j} f\left(z_{j}\right)=W=\sum_{i} f\left(y_{j}\right)+\sum_{j} f\left(z_{j}\right)$ and the series converge absolutely, so that $\sum_{i} f\left(x_{i}\right)=\sum_{i} f\left(y_{j}\right)$.

Let $m$ be a completely additive signed measure. Then the function $f(x):=$ $m(\operatorname{sp}(x)), x \in \mathcal{S}(H)$, is a frame function. Conversely, any frame function $f$ defines a completely additive measure on $\mathcal{L}(H)$. Indeed, let $\left\{x_{i}\right\}$ be an ONB of $M \in \mathcal{L}(H)$. Then the mapping $m(M):=\sum_{i} f\left(x_{i}\right)=W_{M}, M \in \mathcal{L}(H)$, where $W_{M}$ is the weight of $f$ on $M$ is a completely additive measure.

Let $S$ be a linear submanifold of $H$ dense in $H$. A function $f: \mathcal{S}(S) \rightarrow[-\infty, \infty]$ is said to be a frame type function on $H$ if (i) for any ONS $\left\{x_{i}\right\}$ in $S$, either $\left\{f\left(x_{i}\right)\right\}$ is summable or $\sum_{i} f\left(x_{i}\right)=\infty$, and (ii) for any finite-dimensional subspace $K$ of $S$, $f \mid \mathcal{S}(K)$ is a frame function on $K$. To show the relationship between a frame type function $f: \mathcal{S}(S) \rightarrow[-\infty, \infty]$ and a dense submanifold $S$, we will write $f \sim(f, S)$. If we change the latter (i) to (i)' for any ONS $\left\{x_{i}\right\}$ in $S$, either $\left\{f\left(x_{i}\right)\right\}$ is summable or $\sum_{i} f\left(x_{i}\right)=-\infty$, then $-f$ satisfies (i), and vice-versa.

We note that there is a result from theory of series, [Fic, Thm 388, p. 319], Riemann's Rearrangement Theorem, saying that if $\left\{a_{n}\right\}$ is an infinite sequence of real numbers such that $\sum_{n} a_{n}$ is finite but $\sum_{n}\left|a_{n}\right|=\infty$, then for any $a \in[-\infty, \infty]$, there is a rearrangement $\left\{a_{n^{\prime}}\right\}$ of $\left\{a_{n}\right\}$ such that $a=\sum_{n^{\prime}} a_{n^{\prime}}$.

Therefore, let $f$ be a frame type function and $\left\{x_{i}\right\}$ be an ONS in $S$. By the Riemann Rearrangement Theorem, if $\left\{f\left(x_{i}\right)\right\}$ is summable, then $\sum_{i}\left|f\left(x_{i}\right)\right|<\infty$, otherwise we can rearrange $\left\{x_{i}\right\}$ to $\left\{x_{i^{\prime}}\right\}$ such that $\sum_{i^{\prime}} f\left(x_{i}\right)=-\infty$ which is absurd.

It is important to emphasize that frame type functions were originally introduced in DoSh] (cf. Dvu3, Sect 3.2.4]) in a stronger form than our notion, because in DoSh it was supposed that (i) has the form " $\left\{f\left(x_{i}\right)\right\}$ is summable for any ONS $\left\{x_{i}\right\}$ in $S$." We say that a frame type function $f \sim(f, S)$ is finite on $\mathcal{S}(S)$ if, for every $x \in \mathcal{S}(S)$, we have $-\infty<f(x)<\infty$.

We note that if $f \sim(f, S)$ is a finite frame type function, $x \in \mathcal{S}(S)$ and $|\lambda|=1$, then $f(x)=f(\lambda x)$.

It is clear that any frame function on $H$ such that $W_{H}>-\infty$ is a frame type function on $H$. In addition, if $f$ is a frame type function on $H$ with given $S$, then if $K$ is a closed subspace of $H$ such that $S \cap K$ is dense in $K$, then $f_{K}:=f \mid \mathcal{S}(S \cap K)$ is a frame type function on $K$.

Example 2.3. Let $H$ be a complex separable Hilbert space, $\operatorname{dim} H=\aleph_{0}$. 
(i) If $T$ is a Hermitian trace operator, then $f(x)=(T x, x), x \in \mathcal{S}(H)$, is a frame function on $H$.

(ii) If $T_{1}$ is a positive unbounded operator and $T$ is a positive Hermitian trace operator, then $f(x)=\left(T_{1}^{1 / 2} x, T_{1}^{1 / 2} x\right)-(T x, x), x \in D\left(T^{1 / 2}\right)$, is a finite frame type function with $S=D\left(T_{1}^{1 / 2}\right)$, where $D\left(T_{1}^{1 / 2}\right)$ is the domain of definition of $T_{1}^{1 / 2}$.

(iii) Let $M$ be a fixed closed subspace of $H, M \subset H, M \neq H$, let $T_{M}$ be a trace operator on $M$. Then

$$
f(x)= \begin{cases}\left(T_{M} x, x\right) & \text { if } x \in M \\ \infty & \text { otherwise }\end{cases}
$$

is an unbounded frame type function with $S=H$.

(iv) Let $\left\{e_{i}\right\}$ be an ONB and let us define a diagonal operator $T$ on $H$ such that $T e_{i}=(-1)^{i} e_{i}$. Then $T$ is a Hermitian operator defined everywhere on $H$ and it defines a bounded symmetric bilinear form $f(x)=(T x, x)$, $x \in \mathcal{S}(H)$, with $S=H$, that is not a frame type function.

The case (iv) can be generalized as follows. Let $\left\{a_{n}\right\}$ be a bounded sequence of real numbers. We set $a_{n}^{+}=\max \left\{a_{n}, 0\right\}$ and $a^{-}=-\inf \left\{a_{n}, 0\right\}$. Let $\left\{e_{n}\right\}$ be a fixed ONB and we define three bounded Hermitian operators $T, T^{+}$and $T^{-}$on $H$ by $T e_{n}=a_{n} e_{n}, T^{+} e_{n}=a_{n}^{+} e_{n}$, and $T^{-} e_{n}=a_{n}^{-} e_{n}$. Then $T=T^{-}+T^{-}$, and $T^{+}$and $T^{-}$are positive and negative parts of $T$, respectively. Using the Riemann Rearrangement Theorem, we have the following four cases:

(I) $\sum_{n} a_{n}$ is convergent, $\sum_{n}\left|a_{n}\right|<\infty$.

(II) $\sum_{n}^{n} a_{n}$ is convergent, $\sum_{n}^{n}\left|a_{n}\right|=\infty$.

(III) $\sum_{n}^{n} a_{n}=\infty$, and $\sum_{n} a_{n}^{+}=\infty, \sum_{n} a_{n}^{-}<\infty$ or $\sum_{n} a_{n}^{+}<\infty, \sum_{n} a_{n}^{-}=\infty$.

(IV) $\sum_{n} a_{n}^{+}=\infty=\sum_{n} a_{n}^{-}$.

If we set $f(x)=(T x, x), x \in \mathcal{S}(H)$, then in case (I), $f$ is a finite bounded frame function, in cases (II) and (IV) it is no frame type function.

Case (III): Let $s_{n}=a_{1}+\cdots+a_{n}, n \geq 1$. Then $s_{n}=s_{n}^{+}-s_{n}^{-}$, where $s_{n}^{+}=$ $a_{1}^{+}+\cdots+a_{n}^{+}$and $s_{n}^{-}=a_{1}^{-}+\cdots+a_{n}^{-}, n \geq 1$. Then either $\left\{s_{n}^{+}\right\}$is convergent and $\left\{s_{n}^{-}\right\}$is divergent or vice-versa. Let $\left\{x_{i}\right\}$ be an arbitrary ONB of $H$. The first possibility implies $T^{+} \in \operatorname{Tr}(H)$ and $T^{-} \notin \operatorname{Tr}(H)$. Then $-\left(T^{-} x_{i}, x_{i}\right) \leq\left(T x_{i}, x_{i}\right) \leq$ $\left(T^{+} x_{i}, x_{i}\right)$, and this gives $T^{+} \notin \operatorname{Tr}(H)$ which is absurd. Thus we have only the second possibility, i.e., $T^{+} \notin \operatorname{Tr}(H)$ and $T^{-} \in \operatorname{Tr}(H)$.

Let $K>0$ be given. Since $\sum_{i}\left(T^{+} x_{i}, x_{i}\right)=\infty$, there is an integer $n_{0}>0$ such that, for every integer $n \geq n_{0}$, we have $\sum_{i=1}^{n}\left(T^{+} x_{i}, x_{i}\right)>K+\operatorname{tr}\left(T^{-}\right)$so that $\sum_{i=1}^{n}\left(T x_{i}, x_{i}\right)=\sum_{i=1}^{n}\left(T^{+} x_{i}, x_{i}\right)-\sum_{i=1}^{n}\left(T^{-} x_{i}, x_{i}\right)>K+\operatorname{tr}(T)-\sum_{i=1}^{n}\left(T^{-} x_{i}, x_{i}\right)>$ $K$ which yields $\sum_{i}\left(T x_{i}, x_{i}\right)=\infty$.

Now let $\left\{z_{j}\right\}$ be an arbitrary ONS in $H$. We have two cases (i) $\sum_{j}\left(T^{+} z_{j}, z_{j}\right)=\infty$ and (ii) $\sum_{j}\left(T^{+} z_{j}, z_{j}\right)<\infty$. In the case (i), using the same way as that for $\left\{x_{i}\right\}$, we have $\sum_{j}\left(T z_{j}, z_{j}\right)=\infty$, and in the case (ii), we have $\sum_{j}\left(T z_{j}, z_{j}\right)$ is convergent. Consequently, we have proved the following statement:

Proposition 2.4. Let $\left\{a_{n}\right\}$ be a bounded sequence of real numbers, $\left\{e_{n}\right\}$ be a fixed $O N B$ on a Hilbert space $H, \operatorname{dim} H=\aleph_{0}$, and let $T e_{n}=a_{n} e_{n}, n \geq 1$. Then $f(x)=(T x, x), x \in \mathcal{S}(H)$, defines a frame type function if and only if either $\sum_{n}\left|a_{n}\right|<\infty$ or $\sum_{n} a_{n}^{+}<\infty, \sum_{n} a_{n}^{-}=\infty$. The first case implies $f$ is a bounded frame function and the second one implies $f$ is a bounded frame type function. 
It is important to note that if $\operatorname{dim} H>1$ is finite, there are unbounded frame functions on $H$, She, Dvu3, Prop 3.2.4]. If $H$ is infinite-dimensional, then the surprising result DoSh asserts that any finite frame type function $f$ such that $\left\{f\left(x_{i}\right)\right\}$ is summable for any ONS $\left\{x_{i}\right\}$ in $S$ is necessarily bounded, see also Dvu3, Thm 3.2.20].

A frame type function $f$ on $H$ defined on a dense linear submanifold $S$ of $H$ is said to be (i) semibounded if $\inf \{f(x): x \in \mathcal{S}(S)\}>-\infty$, and (ii) bounded if $\sup \{|f(x)|: x \in \mathcal{S}(S)\}<\infty$.

Semibounded frame type functions are connected with semibounded bilinear forms as it follows from the following theorem.

Theorem 2.5. Let $f \sim(f, S)$ be a semibounded finite frame type function on $H$, $\operatorname{dim} H \neq 2$. There is a unique semibounded symmetric bilinear form $t$ with $D(t)=S$ and $f(x)=t(x, x), x \in \mathcal{S}(S)$.

Proof. First, let $H$ be finite-dimensional and $\operatorname{dim} H \neq 2$. Then $H=S$ and $f$ is a frame function. Since $f$ is semibounded, then $f$ is bounded on $\mathcal{S}(H)$. Indeed, let $y$ be any unit vector of $H$ and complete it by unit vectors $x_{1}, \ldots, x_{n}$ to be $\left\{y, x_{1}, \ldots, x_{n}\right\}$ an ONB of $H$. Let $K=\inf \{f(x): x \in \mathcal{S}(H)\}$. Then $|f(y)| \leq$ $W_{H}+n|K|$. By Gleason's Theorem [Dvu3, Thm 3.2.1], there is a unique Hermitian operator $T$ on $H$ such that $f(x)=(T x, x), x \in \mathcal{S}(H)$, and if we set $t(x, x)=(T x, x)$, $x \in H$, then $t$ is a bounded symmetric bilinear form such with $D(t)=S=H$ and $f(x)=t(x, x), x \in \mathcal{S}(H)$.

Now let $\operatorname{dim} H=\infty$. Let $N$ be a finite-dimensional subspace of $S, \operatorname{dim} N \geq 3$. As in the first part of the present proof, $f$ is bounded on $\mathcal{S}(N)$. There is a Hermitian operator $T_{N}: N \rightarrow N$ and a bounded symmetric bilinear form $t_{N}$ on $N \times N$ such that $f(x)=t_{N}(x, x)=\left(T_{N} x, x\right), x \in \mathcal{S}(N)$. We define a bilinear form $t$ on $S \times S$ as follows: Let $x$ and $y$ be two vectors of $S$ and let $N$ be any finite-dimensional subspace of $S, \operatorname{dim} N \geq 3$, containing both $x$ and $y$. Put $t(x, y)=t_{N}(x, y)$. This $t$ is a well-defined bilinear form on $S \times S$ since if $M$ is another finite-dimensional subspace of $S, \operatorname{dim} M \geq 3$, containing $x$ and $y$, then for $Q=M \vee N$, we have $t_{N}(x, y)=t_{Q}(x, y)=t_{M}(x, y)$. It is evident that $f(x)=t(x, x), x \in \mathcal{S}(S)$.

Then $t$ is a semibounded symmetric bilinear form on $H$ with $D(t)=S$. The uniqueness of $t$ follows from the Gleason Theorem.

We note that if $f \sim(f, S)$ is a frame type function such that $\left\{f\left(x_{i}\right)\right\}$ is summable for any ONB $\left\{x_{i}\right\}$ in $S$ and $\operatorname{dim} S \neq 2$, then Theorem 2.5 can be strengthened as follows (cf. Dvu3, Thm 3.2.21]): There is a unique Hermitian trace operator $T$ on $H$ such that

$$
f(x)=(T x, x), \quad x \in \mathcal{S}(S) .
$$

The proof of the following important lemma by Luguvaya-Sherstnev can be found in [LuSh], see also Dvu3, Lem 3.4.2, Cor 3.4.3].

Lemma 2.6 (Lugovaya-Sherstnev). Let $\operatorname{dim} H=3$ and let $f$ be a frame function on $H$. Let there be two orthogonal unit vectors $x$ and $y$ such that $f(x)$ and $f(y)$ are finite. If $W_{H}=\infty$ and $f(z)$ is finite, then $z \in \operatorname{sp}(x, y)$.

Theorem 2.7. Let $\operatorname{dim} H \neq 2$ and $f$ be a semibounded frame function on $H$ such that there is an $O N B\left\{x_{i}\right\}$ with $f\left(x_{i}\right)$ finite for any unit vector $x_{i}$. There is a unique semibounded symmetric bilinear form $t$ with $D(t)$ dense in $H$ such that 


$$
f(x)= \begin{cases}t(x, x) & \text { if } x \in \mathcal{S}(D(t)) \\ \infty & \text { if } x \in \mathcal{S}(H) \backslash \mathcal{S}(D(t)) .\end{cases}
$$

Proof. If $H$ is finite-dimensional, $W_{H}$ and $f$ are finite, the proof follows the same ideas as the first part of the proof of Theorem 2.5 with $D(t)=H$.

Now let $\operatorname{dim} H=\infty$ and let $\left\{x_{i}\right\}$ be an ONB of $H$ such that every $f\left(x_{i}\right)$ is finite. Denote by $D(f):=\{x \in H \backslash\{\mathbf{0}\}: f(x /\|x\|)$ is finite $\} \cup\{\mathbf{0}\}$. Then $x_{i} \in D(f)$ for any $x_{i}$. We assert that $D(f)$ is a linear submanifold of $H$. Indeed, let $x, y \in D(f)$ be two nonzero vectors. Without loss of generality, we can assume $x$ and $y$ are two unit vectors from $D(f)$ that are linearly independent. If $x \perp y$, then clearly $x+y \in \operatorname{sp}(x, y)$. Now let $x \not \perp y$.

Take two vectors $x_{1}, x_{2}$ from the ONB $\left\{x_{i}\right\}$ and set $P=\operatorname{sp}\left(x_{1}, x_{2}\right)$. If $x, y \in P$, then $x+y \in P$ and $x+y \in D(f)$. Let $x \notin P$ and define $M_{1}=\operatorname{sp}\left(x_{1}, x_{2}, x\right)$. We assert the weight $W_{M_{1}}$ of $f$ on $M_{1}$ is finite; if not, by the Lemma LugovayaSherstnev we conclude $x \in P$ which is absurd.

If $y \in M_{1}$, then clearly $x+y \in M_{1}$ and $x+y \in D(t)$. Assume finally $y \notin M_{1}$. Choose a unit vector $z \in M_{1}$ that is orthogonal to $x$. Again by Lemma 2.6, we conclude that $f$ on $M_{2}=\operatorname{sp}(x, y, z)$ has finite weight, so that $x+y \in D(f)$.

Since $D(f)$ contains the ONB $\left\{x_{i}\right\}, S:=D(f)$ is dense in $H$. Since $f \mid S \sim$ $(f \mid S, S)$ is a semibounded finite frame type function on $H$, by Theorem 2.5, there is a unique semibounded symmetric bilinear form $t$ with dense $D(t)=S=D(f)$ such that $f(x)=t(x, x), x \in \mathcal{S}(S)$. Hence, (2.2) holds.

It is clear that if $t$ is a positive symmetric bilinear form with $D(t)$ dense in $H$, then $f(x):=t(x, x), x \in \mathcal{S}(D(t))$, is a finite frame type function on $H$. Thus for positive finite frame type functions and positive symmetric bilinear forms with dense domain there is a one-to-one correspondence. It is worthy of attention that for non-positive bilinear forms such a correspondence is not true, in general, as it follows from Example 2.3(iv), where there is presented a bounded bilinear form not positive that is not a frame type function.

If $t$ is a positive symmetric bilinear form with dense domain, then $f$ defined on $\mathcal{S}(H)$ by $(2.2)$ is not necessarily a frame function. For example, in [Lug1, see also [Dvu3, Thm 3.7.2], it is shown that if $t$ is a singular positive symmetric bilinear form with dense domain, then $f$ defined by $(2.2)$ is not a frame function, but $f \mid D(t)$ is a positive finite frame type function.

We note that according to DoSh, Dvu3, Thm 3.2.20], every finite frame type function $f \sim(f, S)$, such that $\left\{f\left(x_{i}\right)\right\}$ is summable for any ONS $\left\{x_{i}\right\}$ in $S$, is bounded. We do not know whether every (our) finite frame type function is semibounded if $\operatorname{dim} H=\infty$. As it was already mentioned, according to Dvu3, Prop 3.2.4], there are unbounded frame type functions for $H$, $\operatorname{dim} H=n \geq 2$, that are not generated by any bilinear form.

\section{Gleason Measures on $\mathcal{L}(H)$ and Generalized Effect Algebras}

In this section, we study finitely additive measures that are regular. Different classes of measures will be studied from the point of view of generalized effect algebras.

A finitely additive measure $m$ on $\mathcal{L}(H)$ is said to be (i) regular if

$$
m(M)=\sup \{m(P): P \subseteq M, P \in \mathcal{L}(H), \operatorname{dim} P<\infty\}, \quad M \in \mathcal{L}(H),
$$


and (ii) $\mathcal{P}(H)_{1}$-bounded if $\sup \{m(\operatorname{sp}(x): x \in D(m)\}<\infty$, where

$$
D(m):=\{x \in H: m(\operatorname{sp}(x))<\infty\} \cup\{\mathbf{0}\} .
$$

According to Dvu3, $m$ satisfies (i) the $L$-S property (L-S stands for Lugovaja and Sherstnev), if there is a two-dimensional subspace $Q$ of $H$ such that $m(Q)<\infty$, (ii) the density property if $D(m)$ is dense in $H$, and (iii) the $L-S$ density property if both (i) and (ii) hold.

We note that the L-S property entails that $D(m)$ is a dense linear subspace of $H$. If $o$ is the zero measure $o$, i.e. $o(M)=0, M \in \mathcal{L}(H)$, then $o$ is a regular measure with the L-S property. In addition, if $\operatorname{dim} H \neq 2$, then a finitely additive measure $m$ has the L-S density property iff $m$ is $\sigma$-finite.

We denote by $\operatorname{Reg}(H)$ the class of regular measures with the L-S property.

An important relationship between regular finitely additive measures with the L$\mathrm{S}$ property and positive bilinear forms with dense domain is the following result, for the proof see [Dvu3, Lem 3.7.8. Thm 3.7.9]. Before that we remind an important notation: Let $t$ be a bilinear form with domain $D(t)$, let $M \in \mathcal{L}(H)$ be given, and let $P_{M}$ be the orthogonal projector of $H$ onto $M$. We define a new bilinear form $t \circ P_{M}$ as a bilinear form whose domain is the set $D\left(t \circ P_{M}\right):=\left\{x \in H: P_{M} x \in D(t)\right\}$. If this bilinear form is determined by a trace operator $T_{M}^{t}$, we write $t \circ P_{M} \in \operatorname{Tr}(H)$ and $\operatorname{tr}\left(t \circ P_{M}\right):=\operatorname{tr}\left(T_{M}^{t}\right)$.

Theorem 3.1. Let $H$ be a Hilbert space.

(1) Let $t$ be a positive bilinear form such that $D(t)$ is dense in $H$. Then the mapping $m_{t}: \mathcal{L}(H) \rightarrow[0, \infty]$ given by

$$
m_{t}(M)= \begin{cases}\operatorname{tr}\left(t \circ P_{M}\right) & \text { if } t \circ P_{M} \in \operatorname{Tr}(H), \\ \infty & \text { otherwise, }\end{cases}
$$

is a regular finitely additive measure with the $L$-S density property.

(2) Let $m$ be a regular finitely additive measure with the $L$-S density property on $\mathcal{L}(H), \operatorname{dim} H \neq 2$. Then there exists a unique bilinear form $t$ with domain $D(t)=D(m)$ such that (3.1) holds.

Since formula (3.1) is a generalization of the famous Gleason formula (2.1), we call measures expressed by bilinear forms via (3.1) also Gleason measures.

We note that by Theorem 3.1 if $m$ is a regular finitely additive measure with the L-S property, there is a unique positive positive bilinear form $t$ with $D(t)=D(m)$ such that (3.1) holds. In other words, if $x \in D(m)$, we have $t(x, x)=\|x\|^{2} m(\operatorname{sp}(x))$ if $x \neq \mathbf{0}$, otherwise, $t(\mathbf{0}, \mathbf{0})=0$.

On the other hand, if $m$ is a finite positive regular measure on $\mathcal{L}(H)$, then $m$ is completely additive Dvu3, Thm 3.7.2]. For unbounded measures this is not a case, in general. More precisely, any positive singular bilinear form $t$ generates by (3.1) a regular measure that is not completely-additive, see [Dvu3, Thm 3.7.6], Lug2. A criterion when a bilinear form $t$ generates a completely additive measure on $\mathcal{L}(H)$, see [ug2, [Dvu3, Thm 3.7.5]: A positive bilinear form $t$ defines through (3.1) a $\sigma$-finite completely additive measure on $\mathcal{L}(H)$ iff for any $M \in \mathcal{L}(H)$,

$$
\overline{\left(t \circ P_{M}\right)_{r}} \in \operatorname{Tr}(H) \text { implies } t \circ P_{M} \in \operatorname{Tr}(H),
$$

where $\overline{\left(t \circ P_{M}\right)_{r}}$ is the closure of the regular part of $t \circ P_{M}$. 
Proposition 3.2. If $m_{1}, m_{2}$ are regular measures on $\mathcal{L}(H)$, then $m_{1}+m_{2}$ is also a regular measure.

Proof. Given $M \in \mathcal{L}(H)$ and $i=1,2$, there are two sequences $\left\{P_{n}^{1}\right\}$ and $\left\{P_{n}^{2}\right\}$ of finite-dimensional subspaces of $M$ such that $m_{i}(M)=\lim _{n} m_{i}\left(P_{n}^{i}\right)$. Then $m_{i}(M)=$ $\lim _{n} m_{i}\left(P_{n}^{1} \vee P_{n}^{2}\right)$ which entails $\left(m_{1}+m_{2}\right)(M)=m_{1}(M)+m_{2}(M)=\lim _{n} m_{1}\left(P_{n}^{1} \vee\right.$ $\left.P_{n}^{2}\right)+\lim _{n} m_{2}\left(P_{n}^{1} \vee P_{n}^{2}\right)=\lim _{n}\left(m_{1}+m_{2}\right)\left(P_{n}^{1} \vee P_{n}^{2}\right)$ which proves regularity of $m_{1}+m_{2}$.

Proposition 3.2 suggests to study the set of regular Gleason measures also from the point of view of generalized effect algebras.

A partial algebra $(E ; \oplus, 0)$ is called a generalized effect algebra if $0 \in E$ is a distinguished element and $\oplus$ is a partially defined binary operation on $E$ which satisfies the following conditions for any $x, y, z \in E$ :

(GEi) $x \oplus y=y \oplus x$, if one side is defined,

(GEii) $(x \oplus y) \oplus z=x \oplus(y \oplus z)$, if one side is defined,

(GEiii) $x \oplus 0=x$,

(GEiv) $x \oplus y=x \oplus z$ implies $y=z$ (cancellation law),

(GEv) $x \oplus y=0$ implies $x=y=0$.

For every generalized effect algebra $E$, a partial binary operation $\ominus$ and a relation $\leq:=\leq_{\oplus}$ can be defined by

(ED) $x \leq y$ and $y \ominus x=z$ iff $x \oplus z$ is defined and $x \oplus z=y$.

Then $\leq$ is a partial order on $E$ under which 0 is the least element of $E$. A generalized effect algebra with the top element $1 \in E$ is called an effect algebra and we usually write $(E ; \oplus, 0,1)$ for it.

For example, let $G$ be an Abelian po-group, i.e. a group $G=(G ;+, 0)$ endowed with a partial order $\leq$ such that $a \leq b$, then $a+c \leq b+c$ for each $c \in G$. Let $G^{+}:=\{g \in G: g \geq 0\}$ be the positive cone of $G$. Then $\left(G^{+} ; \oplus, 0\right)$ is a generalized effect algebra, where $a \oplus b=a+b, a, b \in G^{+}$. If $u \in G^{+}$is a fixed element, then the interval $[0, u]:=\{g \in G: 0 \leq g \leq u\}$ defines an effect algebra $([0, u] ; \oplus, 0, u)$, where $a \oplus b=a+b, a, b \in[0, u]$, whenever $a+b \leq u$.

In particular, if $\mathcal{B}(H)$ is the class of all Hermitian operators of a Hilbert space $H$, then $\mathcal{B}(H)$ is a po-group, saying $A \leq B$ iff $(A x, x) \leq(B x, x), x \in H$, and $\mathcal{B}(H)^{+}$ is a generalized effect algebra. If $\mathcal{E}(H):=\{A \in \mathcal{B}(H): O \leq A \leq I\}$, where $O$ and $I$ are the zero and identity operators, respectively, then $\mathcal{E}(H)$ is a prototypical example of effect algebras important for Hilbert space quantum mechanics.

A subset $S$ of $E$ is called a sub-generalized effect algebra (sub-effect algebra) of $E$ iff (i) $0 \in S(1 \in S)$, (ii) if out of elements $x, y, z \in E$ such that $x \oplus y=z$ at least two are in $S$, then all $x, y, z \in S$.

The following theorem follows ideas from [RZP], where a structure of linear operators was described.

Theorem 3.3. Let $H$ be an infinite-dimensional complex Hilbert space. Let $\operatorname{Reg}_{f}(H)$ be the set of regular finitely additive measures $m$ on $\mathcal{L}(H)$ with the $L$-S density property such that if $m$ is $\mathcal{P}_{1}(H)$-bounded, then $D(m)=H$. Let us define a partial operation $\oplus$ on $\operatorname{Reg}_{f}(H):$ For $m_{1}, m_{2} \in \operatorname{Reg}_{f}(H), m_{1} \oplus m_{2}$ is defined if and only if $m_{1}$ or $m_{2}$ is $\mathcal{P}_{1}(H)$-bounded or $D\left(m_{1}\right)=D\left(m_{2}\right)$ and then $m_{1} \oplus m_{2}:=m_{1}+m_{2}$. Then $\left(\operatorname{Reg}_{f}(H) ; \oplus, o\right)$ is a generalized effect algebra. 
Proof. Let $m_{1}, m_{2} \in \operatorname{Reg}_{f}(H)$ be measures such that $m_{1} \oplus m_{2}$ is defined. By Theorem 3.1, there are unique positive positive bilinear forms $t_{1}, t_{2}$ with $D\left(t_{1}\right)=$ $D\left(m_{1}\right), D\left(t_{2}\right)=D\left(m_{2}\right)$ such that (3.1) holds. Using [DvJa, Thm 4.1], we have that $t_{1}+t_{2}$ is a densely defined positive bilinear form on $D\left(t_{1}+t_{2}\right)=D\left(t_{1}\right) \cap D\left(t_{2}\right)$. Let $M \in \mathcal{L}(H)$ be such that $m_{1}(M), m_{2}(M)<\infty$. Then $\left(m_{1} \oplus m_{2}\right)(M)=m_{1}(M)+$ $m_{2}(M)=\operatorname{tr}\left(t_{1} \circ P_{M}\right)+\operatorname{tr}\left(t_{2} \circ P_{M}\right)=\operatorname{tr}\left(t_{1} \circ P_{M}+t_{2} \circ P_{M}\right)=\operatorname{tr}\left(\left(t_{1}+t_{2}\right) \circ P_{M}\right)$. If $m_{1}(M)=\infty$ or $m_{2}(M)=\infty$, we have $t_{1} \circ P_{M} \notin \operatorname{Tr}(H)$ or $t_{2} \circ P_{M} \notin \operatorname{Tr}(H)$ which yields $\left(t_{1}+t_{2}\right) \circ P_{M} \notin \operatorname{Tr}(H)$. Therefore, $m_{1} \oplus m_{2}$ is determined by $t_{1}+t_{2}$ in the sense of (3.1) i.e. it is regular and possesses the L-S density property.

(GEi) It follows from the commutativity of the usual sum + .

(GEii) Let $m_{1}, m_{2}, m_{3} \in \operatorname{Reg}_{f}(H)$ such that $\left(m_{1} \oplus m_{2}\right) \oplus m_{3}$ is defined. First, let us assume that $D\left(m_{1}\right)=H$. Then $D\left(m_{1}\right) \cap D\left(m_{2}\right)=D\left(m_{2}\right)$ and $D\left(m_{2}\right)=D\left(m_{3}\right)$ or $D\left(m_{3}\right)=H$. That is, $m_{2} \oplus m_{3}$ is defined with $D\left(m_{2}+m_{3}\right)=D\left(m_{2}\right)$, hence also $m_{1} \oplus\left(m_{2} \oplus m_{3}\right)$ is defined. The same analogy holds for $D\left(m_{2}\right)=H$. If $D\left(m_{1}\right), D\left(m_{2}\right) \neq H$, we have $D\left(m_{1}\right)=D\left(m_{2}\right)$ and then $D\left(m_{3}\right)=D\left(m_{2}\right)$ or $D\left(m_{3}\right)=H$, which yields the existence of $m_{1} \oplus\left(m_{2} \oplus m_{3}\right)$. The equality follows from the associativity of the usual sum + .

(GEiii) It holds since $o \in \operatorname{Reg}_{f}(H)$ is $\mathcal{P}_{1}(H)$-bounded, hence $D(o)=H$.

(GEiv) Let $m_{1} \oplus m_{2}=m_{1} \oplus m_{3}$. By Theorem 3.1] for any $i=1,2,3$, there is a unique positive positive bilinear form $t_{i}$ with $D\left(t_{i}\right)=D\left(m_{i}\right)$ such that (3.1) holds.

Let $m_{1}, m_{2}, m_{3}$ be all $\mathcal{P}_{1}(H)$-bounded. Then $t_{1}(x, y)+t_{2}(x, y)=t_{1}(x, y)+$ $t_{3}(x, y)$ for all $x, y \in H$, which is equivalent to $t_{2}(x, y)=t_{3}(x, y)$ for all $x, y \in H$ hence $t_{2}=t_{3}$, so that $m_{2}=m_{3}$ by Theorem 3.1 .

Let $m_{1}$ be $\mathcal{P}_{1}(H)$-bounded and let $m_{2}$ be not $\mathcal{P}_{1}(H)$-bounded. Then $m_{3}$ has to be not $\mathcal{P}_{1}(H)$-bounded and $D\left(m_{2}\right)=D\left(m_{1}+m_{2}\right)=D\left(m_{1}+m_{3}\right)=D\left(m_{3}\right)$. By the same argument as in the previous case (restricted on $D\left(m_{2}\right)$ ) we have $t_{2}=t_{3}$ and consequently, $m_{2}=m_{3}$.

Let $m_{1}$ be not $\mathcal{P}_{1}(H)$-bounded and let $m_{2}$ be $\mathcal{P}_{1}(H)$-bounded. Then $D\left(m_{1}\right)=$ $D\left(m_{1}+m_{2}\right)=D\left(m_{1}+m_{3}\right)$. For every $x, y \in D\left(t_{1}\right)$, we have $t_{1}(x, y)+t_{2}(x, y)=$ $t_{1}(x, y)+t_{3}(x, y)$. Hence, $t_{2}(x, y)=t_{3}(x, y)$, that is $t_{3}\left|D\left(t_{1}\right)=t_{2}\right| D\left(t_{1}\right)$. Since $t_{2}$ is on $D\left(t_{1}\right)$ bounded, $t_{3}$ is also bounded and by [DvJa, Lem 4.1], $t_{3}$ can be extended on $H$ in a unique way to a symmetric bilinear form, that is $t_{2}=t_{3}$ which implies $m_{2}=m_{3}$.

Let $m_{1}$ and $m_{2}$ be not $\mathcal{P}_{1}(H)$-bounded. Then $D\left(m_{1}\right)=D\left(m_{2}\right)=D\left(m_{1}+m_{2}\right)=$ $D\left(m_{1}+m_{3}\right)$ and in the same way as in the previous case, $t_{3}\left|D\left(t_{1}\right)=t_{2}\right| D\left(t_{1}\right)$. Since $m_{2}$ is not $\mathcal{P}_{1}(H)$-bounded, $m_{3}$ is also not $\mathcal{P}_{1}(H)$-bounded. Because $m_{1} \oplus m_{3}$ is defined, we have $D\left(t_{1}\right)=D\left(t_{3}\right)=D\left(t_{2}\right)$, hence $t_{3}=t_{2}$ and consequently, $m_{3}=m_{2}$.

(GEv) Assume that there is $m_{1}, m_{2} \in \operatorname{Reg}_{f}(H)$ satisfying $m_{1} \oplus m_{2}=o$. Then $D\left(m_{1} \oplus m_{2}\right)=D(o)=H$ and there exist unique positive bilinear forms $t_{1}, t_{2}$ with $D\left(t_{1}\right)=D\left(t_{2}\right)=H$ such that (3.1) holds. Using [DvJa, Thm 4.1], we have $t_{1}=t_{2}=o$ which means $m_{1}=m_{2}=o$.

Theorem 3.4. Let $H$ be a separable infinite-dimensional complex Hilbert space. Let $\operatorname{Reg}_{f}^{\sigma}(H)$ be the set of $\sigma$-additive measures from $\operatorname{Reg}_{f}(H)$. Then $\left(\operatorname{Reg}_{f}^{\sigma}(H)\right.$; $\left.\oplus_{\mid \operatorname{Reg}_{f}^{\sigma}(H)}, o\right)$ is a generalized effect algebra, but $\operatorname{Reg}_{f}^{\sigma}(H)$ is not a sub-generalized effect algebra of the generalized effect algebra $\left(\operatorname{Reg}_{f}(H) ; \oplus, o\right)$.

Proof. Clearly, $o \in \operatorname{Reg}_{f}^{\sigma}(H)$. It is straightforward to show that if a sum $m_{1} \oplus m_{2}$ of two measures from $\operatorname{Reg}_{f}^{\sigma}(H)$ exists in $\operatorname{Reg}_{f}(H)$, it is a measure from $\operatorname{Reg}_{f}^{\sigma}(H)$ as 
well. Let us consider a regular measure $m_{1} \in \operatorname{Reg}_{f}(H)$ given by $m_{1}(M)=\operatorname{dim} M$, $M \in \mathcal{L}(H)$, which is a $P_{1}(H)$-bounded $\sigma$-additive measure and it corresponds to the bilinear form $t_{I}$ corresponding to the identity operator $I$ on $H$, i.e. $t_{I}(x, x)=$ $(I x, x)=(x, x), x \in H$. Let $m_{2} \in \operatorname{Reg}_{f}(H)$ be a finitely additive measure given by (3.1) for some positive singular bilinear form $s$ with $D(s)=H$, hence by Dvu3, Thm 3.7.2], $m_{2}$ is not $\sigma$-additive. Since $D(s)=H$, it holds $s \circ P_{M} \in \operatorname{Tr}(H)$ if $\operatorname{dim} M<\infty$. Moreover, $t_{I} \circ P_{M}=P_{M} \in \operatorname{Tr}(H)$ iff $\operatorname{dim} M<\infty$ which gives $\left(s+t_{I}\right) \circ P_{M} \in \operatorname{Tr}(H)$ iff $\operatorname{dim} M<\infty$. By [Sim, Cor 2.3], we have $\overline{\left(s+t_{I}\right)_{r}}=t_{I}$ and using (3.2) or [Dvu3, Thm 3.7.5], $m_{1} \oplus_{\mid \operatorname{Reg}_{f}^{\sigma}} m_{2}:=m_{1} \oplus m_{2}=m_{1}+m_{2}$ is a $\sigma$-additive measure. Which means that $\operatorname{Reg}_{f}^{\sigma}(H)$ is not a sub-generalized effect algebra of $\operatorname{Reg}_{f}(H)$ because $m_{1}, m_{1} \oplus m_{2} \in \operatorname{Reg}_{f}^{\sigma}(H)$ but $m_{2} \notin \operatorname{Reg}_{f}^{\sigma}(H)$.

Remark 3.5. Let $H$ be an infinite-dimensional complex Hilbert space. Let $\operatorname{Reg}_{f}^{c}(H)$ be the set of completely additive measures from $\operatorname{Reg}_{f}(H)$. Then by [Dvu3, Thm 3.6.3] $\operatorname{Reg}_{f}^{c}(H)=\operatorname{Reg}_{f}^{\sigma}(H)$, which yields $\left(\operatorname{Reg}_{f}^{c}(H) ; \oplus, o\right)$ is a generalized effect algebra.

Using Theorem 2.5] and Theorem 2.7, we can extend the previous results also for frame type functions. For any two frame type function $f_{1}, f_{2}$ on $H$, their usual sum $f(x):=f_{1}(x)+f_{2}(x)$ for all $x \in \mathcal{S}\left(S_{1}\right) \cap \mathcal{S}\left(S_{2}\right)$ is again a frame type function, whenever $S_{1} \cap S_{2}$ is dense in $H$.

A frame function $o_{f}$ is defined by $o_{f}(x)=0$ for all $x \in \mathcal{S}(H)$.

Theorem 3.6. Let $H$ be an infinite-dimensional complex Hilbert space. Let $\mathrm{Ftf}(H)$ be the set of positive finite frame type functions $f \sim(f, S)$ on $H$ such that whenever $f$ is bounded, then $S=H$. Let us define a partial operation $\hat{\oplus}$ on $\operatorname{Ftf}(H)$ : For $f_{1}, f_{2} \in \operatorname{Ftf}(H), f_{1} \hat{\oplus} f_{2}$ is defined if and only if $f_{1} \sim\left(f, S_{1}\right)$ or $f_{2} \sim\left(f, S_{2}\right)$ is bounded or $S_{1}=S_{2}$ and then $f_{1} \hat{\oplus} f_{2}:=f_{1}+f_{2}$. Then $\left(\operatorname{Ftf}(H) ; \hat{\oplus}, o_{f}\right)$ is a generalized effect algebra.

Proof. By Theorem 2.5, there is a one-to-one correspondence between positive finite frame type functions and positive bilinear forms given by $f(x)=t(x, x)$ for $x \in D(t)$ and $S=D(t)$. A positive frame type function $f$ is bounded iff the corresponding bilinear form $t$ is bounded iff the corresponding finitely additive measure $m$ is bounded, and clearly $S=D(t)=D(m)$. Hence, the sets $\operatorname{Ftf}(H)$ and $\operatorname{Reg}_{f}(H)$ from Theorem 3.4 are in a one-to-one correspondence.

For any two frame type functions $f_{1}, f_{2} \in \operatorname{Ftf}(H), f_{1} \hat{\oplus} f_{2}$ is defined if and only if for finitely additive measures $m_{1}, m_{2}$, given by bilinear forms $t_{1}(x, x):=$ $f_{1}(x), t_{2}(x, x):=f_{2}(x), m_{1} \oplus m_{2}$ is defined and then $\left(f_{1} \hat{\oplus} f_{2}\right)(x)=\left(m_{1} \oplus m_{2}\right)(\operatorname{sp}(x))$. That is, the proof follows the same arguments as the proof of Theorem 3.3 .

Remark 3.7. Let $f \sim(f, S)$ be a frame type function on $H$. If the function $\bar{f}$ given by $\bar{f}(x):=f(x)$ for all $x \in S$ and $f(x):=\infty$ otherwise is a frame function on $H$, then we say that $f$ induces a frame function $\bar{f}$.

Theorem 3.8. Let $H$ be an infinite-dimensional complex Hilbert space. Let $\mathrm{Ff}(H) \subseteq$ $\operatorname{Ftf}(H)$ be the set of all frame type functions $f$ on $H$ which induce a frame function $\bar{f}$. Then $\operatorname{Ff}(H)$ is a subset of $\operatorname{Ftf}(H)$ but it is not a sub-generalized effect algebra of $\left(\operatorname{Ftf}(H) ; \hat{\oplus}, o_{f}\right)$, but $\left(\operatorname{Ftf}(H) ; \hat{\oplus}_{\mid \operatorname{Ftf}(H)}, o_{f}\right)$ is a generalized effect algebra on its own. 
Proof. A restriction of any finite frame function $\bar{f}$ on $S=\{x \in H \mid f(x)<\infty\}$ is a finite frame type function $f \sim(f, S)$, and on the other hand, $f$ induces $\bar{f}$. Since there is a one-to-one correspondence between the set of positive frame functions $\operatorname{Ff}(H)$ and the set of completely additive measures $\operatorname{Reg}_{f}^{c}(H)$, the theorem follows from Theorem 3.4 and Remark 3.5 .

\section{Monotone COnVERGEnCE Properties of $\sigma$-ADditive MeAsures}

In the section, we will study some monotone Dedekind upwards (downwards) $\sigma$-complete properties of generalized effect algebras of regular measures.

In Dvu3, Thm 3.10.1], there was proved an analogue of the Nikodým convergence theorem which says following:

Theorem 4.1. Let $\left\{m_{n}\right\}$ be a sequence of finite signed $\sigma$-additive measures on $\mathcal{L}(H)$ of a Hilbert space $H$. If there is a finite limit $m(M)=\lim _{n \rightarrow \infty} m_{n}(M)$ for any $M \in \mathcal{L}(H)$, then $m$ is a finite signed measure on $\mathcal{L}(H)$, and $\left\{m_{n}\right\}$ is uniformly $\sigma$-additive with respect to $n$.

We say that a generalized effect algebra $E$ is (i) monotone Dedekind upwards $\sigma$ complete if, for any sequence $x_{1} \leq x_{2} \leq \cdots$, which is dominated by some element $x_{0}$, i.e. each $x_{n} \leq x_{0}$, the element $x=\bigvee_{n} x_{n}$ is defined in $E$ (we write $\left\{x_{n}\right\} \nearrow x$ ), (ii) monotone Dedekind downwards $\sigma$-complete if, for any sequence $x_{1} \geq x_{2} \geq \cdots$, the element $x=\bigwedge_{n} x_{n}$ is defined in $E$ (we write $\left\{x_{n}\right\} \searrow x$ ). If $E$ is an effect algebra, both later notions are equivalent. In addition, we say that a generalized effect algebra $E$ is upwards directed if, given $a_{1}, a_{2} \in E$, there is $a \in E$ such that $a_{1}, a_{2} \leq a$.

Theorem 4.2. Let $H$ be an infinite-dimensional complex Hilbert space. Then the generalized effect algebra $\left(\operatorname{Reg}_{f}(H) ; \oplus, o\right)$ is monotone downwards $\sigma$-complete, but it is not Dedekind monotone upwards $\sigma$-complete.

Proof. This holds since $\left(\operatorname{Reg}_{f}(H) ; \oplus, o\right)$ is isomorphic to the generalized effect algebra of all positive bilinear forms on $H$, which by [DvJa, Thm 5.2] has the mentioned properties.

Theorem 4.3. Let $H$ be an infinite-dimensional complex Hilbert space. The generalized effect algebra $\left(\operatorname{Reg}_{f}^{\sigma}(H) ; \oplus, o\right)$ is neither monotone downwards $\sigma$-complete, nor Dedekind monotone upwards $\sigma$-complete.

Proof. Let us consider the complex Hilbert space $H=L^{2}(0,1)$ and the following sequence of bilinear forms $\left\{s_{n}\right\}_{n \in \mathbb{N}}$ given by

$$
s_{n}(u, u):=\left(1+\frac{1}{n}\right) \int_{0}^{1}\left|u^{\prime}(x)\right|^{2} \mathrm{~d} x+|u(0)|^{2}+|u(1)|^{2},
$$

where $u^{\prime}$ is derivative of $u \in L^{2}[0,1]$. Let

$$
\begin{gathered}
s(u, u):=\int_{0}^{1}\left|u^{\prime}(x)\right|^{2} \mathrm{~d} x+|u(0)|^{2}+|u(1)|^{2}, \\
\hat{s}(u, u):=\int_{0}^{1}\left|u^{\prime}(x)\right|^{2} \mathrm{~d} x, \\
s_{0}(u, u):=|u(0)|^{2}+|u(1)|^{2} .
\end{gathered}
$$


Then $s, \hat{s}$ and $s_{n}$ are closed bilinear forms for all $n \in \mathbb{N}$. According to [Dvu1, Prop 3.7.4], measures $m_{s}, m_{\hat{s}}$ and $m_{s_{n}}$ induced by these bilinear forms via (3.1) are $\sigma$-additive (due to their regularity also completely additive). Also $s-s_{n}, \hat{s}-s_{n}$ are closed bilinear forms, which means that $m_{s}, m_{\hat{s}} \leq_{\sigma} m_{s_{n}}$ for all $n \in \mathbb{N}$, where $\leq_{\sigma}$ is the partial order in the generalized effect algebra $\operatorname{Reg}_{f}^{\sigma}(H)$ induced by the partial addition in it via (ED). It holds $m_{\hat{s}} \leq_{\sigma} m_{s}$ in the generalized effect algebra $\operatorname{Reg}_{f}(H)$, but since $s-\hat{s}$ is a singular bilinear form, the corresponding measure $m_{s-\hat{s}}$ is not $\sigma$-additive hence $m_{\hat{s}} \not_{\sigma} m_{s}$. Since $m_{s}=\bigvee_{n \in \mathbb{N}} m_{s_{n}}$ in the generalized effect algebra $\operatorname{Reg}_{f}(H)$, the sequence $\left\{m_{s_{n}}\right\}_{n \in \mathbb{N}}$ has no infimum in $\operatorname{Reg}_{f}^{\sigma}(H)$. By

DvJa, Lemma 5.1], $\operatorname{Reg}_{f}^{\sigma}(H)$ is not a Dedekind upwards $\sigma$-complete generalized effect algebra.

\section{REFERENCES}

[Fic] G.M. Fichtengol'c, Kurs differencial'nogo i integral'nogo isčislenia", Fizmatgiz, Moskva, 1962 (in Russian).

[DoSh] S.V. Dorofeev, A.N. Sherstnev, Frame-type functions and their applications, Izv. vuzov matem. No. 4 (1990), 23-29 (in Russian). English translation: Sov. Math. 34 No. 4, (1990), 25-31.

[Dvu1] A. Dvurečenskij, Signed states on a logic, Math. Slovaca 28 (1978), 33-40.

[Dvu2] A. Dvurečenskij, Converse of the Eilers-Horst theorem, Inter. J. Theor. Phys. 26 (1987), 609-612.

[Dvu3] A. Dvurečenskij, "Gleason's Theorem and its Applications", Mathematics and its Applications, Vol. 60. Kluwer Acad. Publ., Dordrecht/Ister Science, Bratislava, 1993.

[DvJa] A. Dvurečenskij, J. Janda, On bilinear forms from the point of view of generalized effect algebras, Found. Phys. 43 (2013), 1136-1152. DOI: 10.1007/s10701-013-9736-2

[DvPu] A. Dvurečenskij, S. Pulmannová, "New Trends in Quantum Structures", Kluwer Academic Publ., Dordrecht, Ister Science, Bratislava, 2000, 541 + xvi pp.

[FoBe] D.J. Foulis, M.K. Bennett, Effect algebras and unsharp quantum logics, Found. Phys. 24 (1994), 1331-1352.

[Gle] A.M. Gleason, Measures on the closed subspaces of a Hilbert space, J. Math. Mech. 6 (1957), 885-893.

[Hal] P.R. Halmos, "Introduction to Hilbert Space and the Theory of Spectral Multiplicity", Chelsea Publ. Co., New York, 2nd ed. 1957.

[Lug1] G.D. Lugovaya, Bilinear forms defining measures on projectors, Izv. Vyssh. Uchebn. Zaved., Mat. No. 2249 (1983), 88-88 (in Russian). English translation: Sov. Math. 27 (1983), 102-102.

[Lug2] G.D. Lugovaya, On a construction of unbounded measures on projectors of Hilbert space, Issled. po priklad. matem., Izdat. Kazan Univ. (1984), 202-205 (in Russian). English translation: J. Sov. Math. 44 No. 5 (1089), 711-713.

[LuSh] G.D. Lugovaya, A.N. Sherstnev, On the Gleason theorem for unbounded measures, Izv. vuzov matem. No. 2 (1980), 30-32 (in Russian). English translation: Sov. Math. 24 No. 12 (1980), 35-38.

[RZP] Z. Riečanová, M. Zajac, S. Pulmannová, Effect algebras of positive linear operators densely defined on Hilbert spaces, Rep. Math. Phys. 68 (2011), 261-270.

[She] A.N. Sherstnev, On a notion of a charge in noncommutative scheme of measure theory, in: "Veroj. metod i kibern.", Kazan, No. 10-11 (1974), pp. 68-72 (in Russian).

[Sim] B. Simon, A canonical decomposition for quadratic forms with applications for monotone convergence theorems, J. Funct. Analysis 28 (1978), 377-385. 